\title{
Síntese por reação por combustão de nanopós de hexaferrita de estrôncio dopada com cromo
}

\section{(Synthesis of chromium-doped strontium hexaferrite nanopowders by combustion reaction)}

\author{
W. S. Castro ${ }^{1}$, R. R. Corrêa ${ }^{2}$, A. J. A. de Oliveira ${ }^{3}$, R. H. G. A. Kiminami ${ }^{1}$ \\ ${ }^{l}$ Departamento de Engenharia de Materiais, Universidade Federal de S. Carlos, Rod. Washington Luiz, km 235, \\ C.P. 676, S. Carlos, SP 13565-905 \\ ${ }^{2}$ Departamento de Eletroeletrônica, Instituto Federal de Educação, Ciência e Tecnologia do Maranhão, \\ Av. Getúlio Vargas 4, Monte Castelo, S. Luís, MA 65030-005 \\ ${ }^{3}$ Departamento de Física, Universidade Federal de S. Carlos, Rod. Washington Luiz, km 235, S. Carlos, SP \\ $13565-905$
}

\begin{abstract}
Resumo
Nanopós de hexaferrita de estrôncio dopadas com cromo foram sintetizadas por reação por combustão. A influência do $\mathrm{Cr}^{3+}$ nas proporções de $\mathrm{x}=0,0,2,0,3$ e 0,4 no sistema $\operatorname{SrCr}_{\mathrm{x}} \mathrm{Fe}_{12-\mathrm{x}} \mathrm{O}_{19}$ foi investigada. Os pós foram preparados de acordo com o conceito da química dos propelentes, aquecidos em placa convencional. Os pós sintetizados foram caracterizados por difratometria de raios X, microscopia eletrônica de varredura e medidas magnéticas. Os resultados mostraram que foi possível obter nanopós de hexaferrita de estrôncio dopada com cromo, que a presença da fase secundária $\left(\mathrm{SrFe}_{2} \mathrm{O}_{5}\right)$ diminui com o aumento da adição de cromo, e que a proporção de $\mathrm{x}=0,4$ de cromo favoreceu $\mathrm{o}$ aumento da magnetização de saturação.
\end{abstract}

Palavras-chave: nanohexaferritas, hexaferrita de estrôncio, cromo, síntese, reação por combustão.

\begin{abstract}
Chromium-doped strontium hexaferrite powder was prepared by combustion reaction. The influence of the Cr ${ }^{3+}$ concentration on the $\mathrm{SrCr}_{x} \mathrm{Fe}_{12-x} \mathrm{O}_{19}$ system was investigated. The powder was prepared according to the concept of propellant chemistry and heated on a conventional plate. The resulting material was characterized by $X$-ray diffraction, scanning electron microscopy and magnetic measurements. The results indicate that chromium-doped hexaferrite nanopowders were obtained successfully. The presence of the secondary phase ( $\left.\mathrm{SrFe}_{2} \mathrm{O}_{5}\right)$ tended to decrease with increasing chromium content and the ratio of $x=0.4$ of chromium enhanced the material's saturation magnetization. Keywords: nano-hexaferrites, strontium hexaferrite, chromo, synthesis, combustion reaction.
\end{abstract}

\section{INTRODUÇÃO}

As hexaferritas de estrôncio ( $\mathrm{SrM}$ ) são cerâmicas ferrimagnéticas, intensamente utilizadas como magnetos permanentes $[1,2]$, e tem como principais características: elevada anisotropia magneto cristalina, altos valores de magnetização e temperatura de Curie, resistência ao calor e à corrosão, excelente estabilidade química e baixa densidade [3]. As propriedades elétricas e magnéticas das ferritas SrM, estão diretamente relacionadas com sua microestrutura, e esta, pode ser modificada e até mesmo controlada, pelas condições de síntese, como os tipos e variáveis de processamento e também pela introdução de dopantes.

Apesar do método de mistura de óxido cerâmico ser relativamente simples, é o mais utilizado industrialmente, porém apresenta desvantagens inerentes, como: impurezas acrescidas durante o processo de moagem, baixo controle da composição, baixa homogeneidade química, tamanho excessivamente grosseiro das partículas, comprometendo a microestrutura e propriedades finais desses materiais $[4,5]$. Para contornar os problemas advindos do método de mistura de óxidos, métodos químicos em escala de laboratório, tais como co-precipitação [6], hidrotérmica [7], reação por combustão [8, 9], sol-gel [11], Pechini [12], autocombustão [13], entre outros, foram desenvolvidos, visando obter principalmente o controle da homogeneidade química, morfológica e granulométrica dos pós, garantindo, assim, o controle da microestrutura e propriedades finais do material. O método de reação por combustão vem se destacando nos últimos anos, devido principalmente á facilidade de sua aplicação, ao custo relativamente baixo, ao controle da homogeneidade química, por requerer poucas etapas e curto 
tempo de duração [8-10]. Este método utiliza a química dos propelentes e explosivos para promover uma reação de uma mistura redox, contendo os íons metálicos de interesse como oxidantes e um combustível, como redutor [8]. Para determinar rapidamente a estequiometria de um sistema com mais de dois componentes, para uma reação de combustão, basta calcular a relação entre a valência total dos oxidantes e do combustível, possibilitando ter uma mistura estequiométrica quando o valor do coeficiente estequiométrico $\Phi_{\varepsilon}$, for igual a 1 [10], isto é:

$$
\Phi_{\varepsilon}=\frac{(\text { coef } \text {.doscomponentesoxidantesxvalencia) }}{(-1) \Sigma \text { coef } \text {.doselementosredutoresxvalencia }}=1
$$

A síntese de reação por combustão é inicializada por uma fonte externa, fazendo com que uma reação exotérmica autossustentável muito rápida aconteça, resultando em um produto final (pó), em um curto período de tempo. Assim, neste contexto este trabalho tem como objetivo a síntese por reação de combustão e caracterização de nanopós de hexaferritas de estrôncio dopadas com cromo.

\section{MATERIAIS E MÉTODOS}

A síntese dos pós do sistema $\mathrm{SrCrxFe}_{12}-\mathrm{xO}_{19}, \mathrm{x}=0,0,2$, $0,3,0,4$ e $0,5 \mathrm{~mol} \% \mathrm{Cr}^{3+}$, foi feita pelo método de reação de combustão em placa quente com [ $\left(\mathrm{Fe}\left(\mathrm{NO}_{3}\right)_{3} \cdot 9 \mathrm{H} 2 \mathrm{O}\right]$, $\left[\mathrm{Sr}\left(\mathrm{NO}_{3}\right)_{2}\right], \mathrm{e}\left[\mathrm{Cr}\left(\mathrm{NO}_{3}\right)_{3} \cdot 9 \mathrm{H}_{2} \mathrm{O}\right]$ como reagentes precursores e fontes de cátions, e a ureia $\left[\mathrm{CO}\left(\mathrm{NH}_{2}\right)_{2}\right]$ como agente redutor, em um cadinho de sílica vítrea como recipiente. A mistura inicial (composição estequiométrica) foi calculada de acordo com as valências dos elementos reativos, de modo a favorecer a relação oxidante/combustível $=1$, de acordo com a química dos propelentes [10]. As reações de combustão ocorreram a $500{ }^{\circ} \mathrm{C}$ e foram monitoradas por um pirômetro de infravermelho de alto desempenho Marathon MM2ML controlado por microcomputador. As amostras foram designadas "sem", SrM0,2, SrM0,3, SrM0,4 e SrM0,5 para os sistemas com $\mathrm{x}=0,0,2,0,3$, 0,4 e $0,5 \mathrm{~mol}^{2} \mathrm{Cr}^{3+}$, respectivamente. Os pós resultantes apresentaram estrutura de flocos porosos, pouco volumosos e de coloração marrom escuro; posteriormente foram desaglomerados em almofariz de ágata, peneirados em malha 325 mesh $(44 \mu \mathrm{m})$. Os pós foram então calcinados a $700{ }^{\circ} \mathrm{C}$ e $1000{ }^{\circ} \mathrm{C}$ com taxa de aquecimento $10{ }^{\circ} \mathrm{C} / \mathrm{min}$ visando à cristalização da fase hexaferrita sem presença de fases secundárias, e depois submetidos à caracterização. A análise de difração de raios $\mathrm{X}$ foi feita em um difratômetro de raios X Siemens D5000 com radiação Cuk $\alpha$. Os difratogramas foram obtidos a $25^{\circ} \mathrm{C}$ na faixa $2 \theta$ de 10 a $70^{\circ}$. A morfologia dos pós foi analisada por meio de microscopia eletrônica de varredura em um microscópio Philips XL30 FEG. Para a determinação das propriedades magnéticas foram realizadas à temperatura ambiente medidas de magnetização em função do campo magnético em um magnetômetro de amostra vibrante VSM PAR 4500 acoplado a um eletro-ímã com campo máximo de até $8 \mathrm{kOe}$.

\section{RESULTADOS E DISCUSSÃO}

As análises das características dos nanopós sintetizados por reação de combustão em placa quente foram feitas em função da concentração do íon $\mathrm{Cr}^{3+}$ no sistema $\mathrm{SrCr}_{x} \mathrm{Fe}_{12-\mathrm{x}} \mathrm{O}_{19}$, $\mathrm{x}=0,0,2,0,3$ e $0,4 \mathrm{~mol}^{\%} \mathrm{Cr}^{3+}$. Foi constatado em todas as composições que a temperatura das reações por combustão alcançou picos entre 890 a $950{ }^{\circ} \mathrm{C}$, tendendo a aumentar a ignição em função do aumento da concentração de $\mathrm{Cr}^{3+}$ no sistema $\mathrm{SrCr}_{x} \mathrm{Fe}_{12-\mathrm{x}} \mathrm{O}_{19}$. O fato de o cromo tender a elevar a temperatura da síntese deve ser provavelmente causado pela razão de o calor de formação do cromo $(-1139,7 \mathrm{~kJ} /$ mol) ser inferior ao de formação do ferro $(-824,2 \mathrm{~kJ} / \mathrm{mol})$, o que necessita gerar maior temperatura para ocorrer completa formação da fase. Esse comportamento já foi reportado no estudo do sistema de nanoferritas de níquel dopadas com cromo por reação de combustão em forno de micro-ondas [14].

A Fig. 1 apresenta os difratogramas de raios $\mathrm{X}$ dos pós obtidos por reação de combustão em placa quente e após calcinação a 700 e $1000{ }^{\circ} \mathrm{C}$. Os nanopós apresentaram a formação da fase cristalina da hexaferrita de estrôncio (JCPDF 80-1198) e presença de traços de fase secundária de $\mathrm{SrFe}_{2} \mathrm{O}_{5}$ (JCPDF 37-1320). Estes resultados confirmam a forte influência da formação desta fase com a temperatura e tempo de reação [15], limitando a calcinação a $1000{ }^{\circ} \mathrm{C}$ para evitar deterioração das propriedades magnéticas.

A Fig. 2 apresenta os difratogramas de raios X dos pós de hexaferrita de estrôncio sem e com cromo após calcinação a $1000{ }^{\circ} \mathrm{C} / 1 \mathrm{~h}$. Os difratogramas mostram que em todas as amostras calcinadas a $1000{ }^{\circ} \mathrm{C} / 1 \mathrm{~h}$ houve a fase hexaferrita de estrôncio com estrutura hexagonal da magnetoplumbita, e presença de traços de fase secundária $\left(\mathrm{SrFe}_{2} \mathrm{O}_{5}\right)$, sendo a mesma reduzida com o aumento da concentração de $\mathrm{Cr}^{3+}$. Esses resultados confirmam a forte influência do cromo, indicando que o aumento da concentração de $\mathrm{Cr}^{3+}$ favoreceu

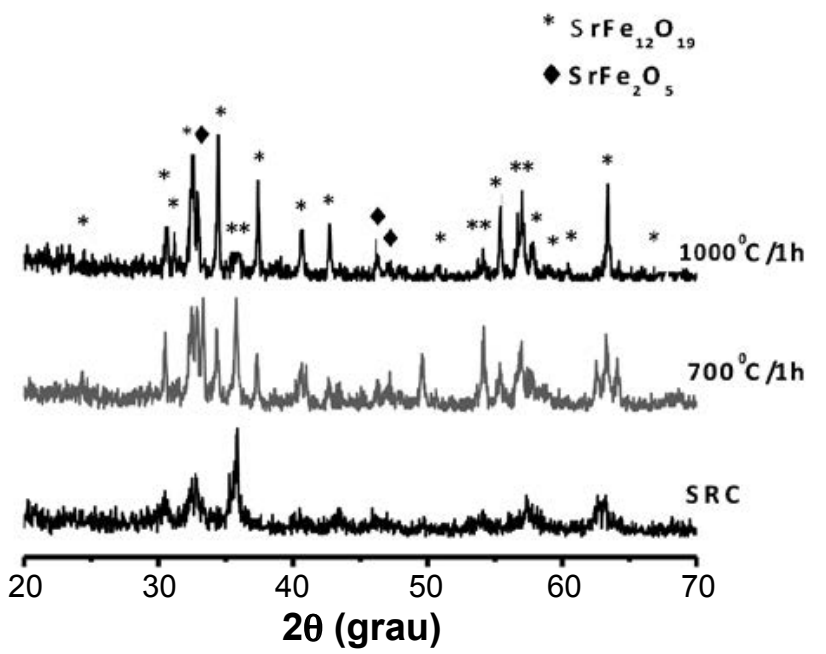

Figura 1: Difratogramas dos pós de hexaferritas de estrôncio, obtidos da síntese de reação por combustão, e calcinados a 700 e $1000{ }^{\circ} \mathrm{C}$ por $1 \mathrm{~h}$.

[Figure 1: XRD patterns of strontium hexaferrite powders synthesized by combustion reaction and calcined at 700 and $1000^{\circ} \mathrm{C}$ for $1 \mathrm{~h}$.] 


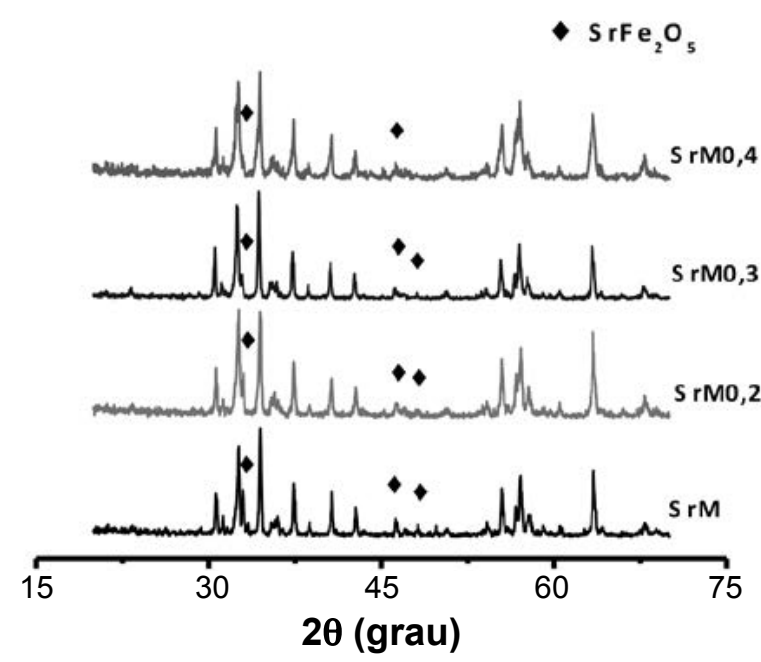

Figura 2: Difratogramas de raios $\mathrm{X}$ da hexaferrita de estrôncio dopada com cromo e calcinadas a $1000{ }^{\circ} \mathrm{C} / 1 \mathrm{~h}$.

[Figure 2: XRD patterns of chromium-doped strontium hexaferrite powders calcined at $1000^{\circ} \mathrm{C} / 1 \mathrm{~h}$.]

ao aumento da temperatura, levando à formação de nanopós com alta cristalinidade da fase hexaferrita de estrôncio, com traços da fase $\left(\mathrm{SrFe}_{2} \mathrm{O}_{5}\right)$.

A Tabela I apresenta os resultados das características dos nanopós sintetizados por reação de combustão. $\mathrm{O}$ aumento da concentração de $\mathrm{Cr}^{3+}$ no sistema da hexaferrita de estrôncio levou a uma redução no tamanho de cristalito. Estes resultados demonstram o efeito inerente da adição do cromo nas características estruturais das hexaferritas de estrôncio e a consequente alteração nos parâmetros de temperatura.

As Figs. 3a, b, c e d apresentam as micrografias para os pós de hexaferrita de estrôncio preparados por reação de combustão em placa quente. As nanopartículas dos quatro sistemas são inferiores a $50 \mathrm{~nm}$, que favoreceu a formação de aglomerados moles de nanopartículas.

Em todos os sistemas estudados as nanopartículas apresentaram morfologia e tamanho uniformes, constituídas apenas por nanopartículas primárias aglomeradas. É sabido que quanto menor o tamanho de partículas, maior a sua área superficial, o que gera força motriz para favorecer o aumento do estado de aglomeração e/ou agregação [16]. Experimentalmente (no processo de peneiramento), quanto maior a concentração de $\mathrm{Cr}^{3+}$ no sistema, mais friável, ou seja, mais fácil foi a desaglomeração do pó. Por meio dessas micrografias, pode-se então observar que para todas

Tabela I - Tamanho de cristalito das amostras sintetizadas por reação por combustão.

[Table I - Crystallite size of powders obtained by combustion reaction.].

\begin{tabular}{cc}
\hline Amostras & Tamanho de cristalito $(\mathrm{nm})$ \\
\hline SrM & 47,3 \\
SrM0,2 & 47,3 \\
SrM0,3 & 43,3 \\
SrM0,4 & 38,1 \\
\hline
\end{tabular}
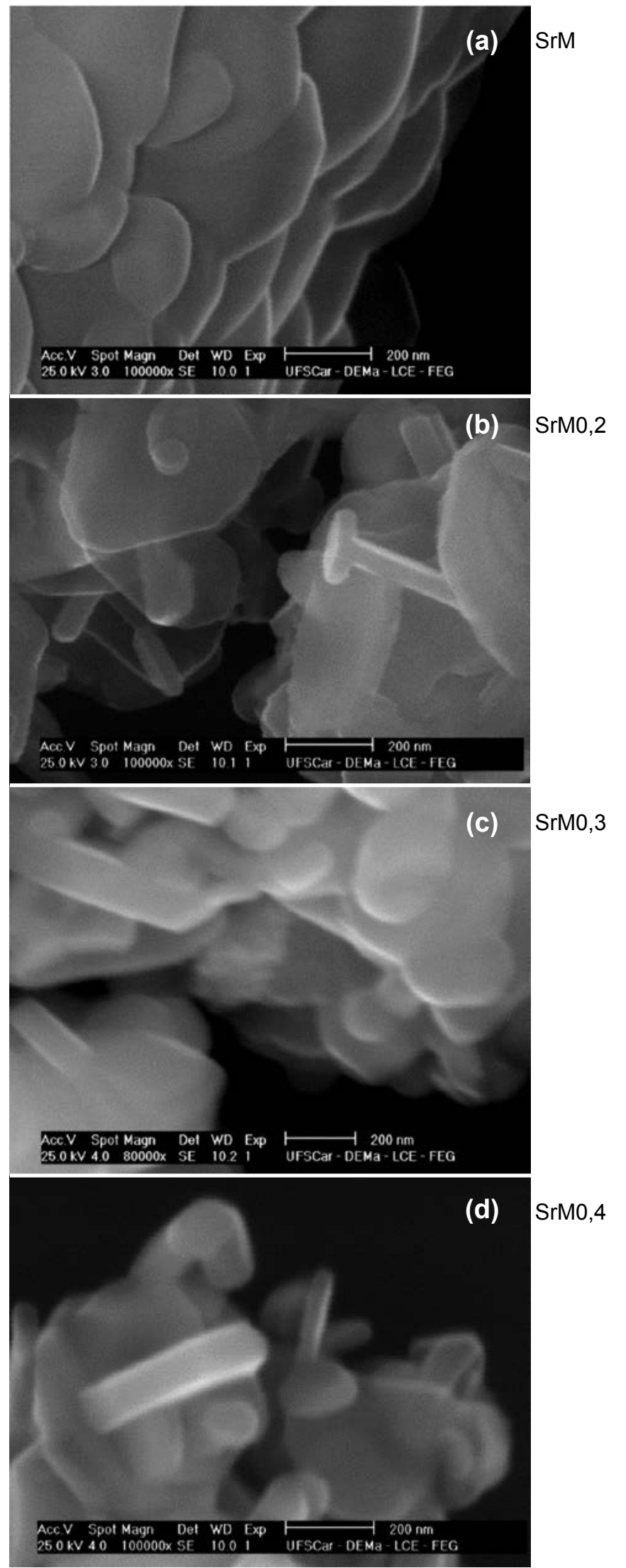

SrM0,4

Figura 3: Micrografias obtidas em microscópio eletrônico de varredura das amostras de hexaferritas de estrôncio dopadas com cromo.

[Figure 3: SEM micrographs of samples of chromium-doped strontium hexaferrite.] 
as composições houve a formação de aglomerados moles (fácil desaglomeração) em forma de plaquetas hexagonais irregulares e um aspecto constituído de partículas bastante finas, e que o estado de aglomeração tornou-se mais mole à medida que se elevou a concentração de íons $\mathrm{Cr}^{3+}$ na hexaferrita de estrôncio.

A Fig. 4 apresenta as curvas de magnetização como função do campo magnético para os sistemas preparados por reação de combustão. As amostras apresentam uma larga histerese. Conforme a Fig. 3, as amostras estudadas apresentam tamanho de grão de $\sim 50 \mathrm{~nm}$, levando a que todas amostras apresentassem campos coercivos semelhantes, entre 3,9 e 4,9 kOe. Nas hexaferritas de estrôncio, conforme aumentou a concentração de cromo na composição, houve um crescimento da magnetização de saturação, sendo máxima para a amostra com $\mathrm{x}=0,4$. $\mathrm{O}$ fato que as curvas de magnetização apresentaram um loop amplo é uma evidência de que a magnetização não foi na direção de fácil magnetização. Além disso, a magnetização apresenta uma pequena inflexão entre 0 e $1 \mathrm{kOe}$, que corrobora com essa hipótese. Tal inflexão surge justamente devido a energia extra que se faz necessária para alinhar os momentos magnéticos quando o campo não está na direção do eixo fácil [17].

A Tabela II mostra os parâmetros magnéticos (campo coercivo, magnetização de saturação, magnetização remanente e perda por histerese) obtidos a partir das curvas $\mathrm{M} \times \mathrm{H}$.

Comparando os sistemas com $\mathrm{x}=0,0,0,2$ e 0,3 mol de cromo, verifica-se uma pequena redução na magnetização de saturação. Porém, comparado com o sistema 0,4 , verificase um pequeno aumento. Isso indica que a substituição de 0,2 a $0,3 \mathrm{~mol}$ de cromo por ferro foi adequada, pois não foi verificada uma perda drástica nessas redução na magnetização, confirmando características ferrimagnéticas em todas as amostras. Estes resultados corroboram com

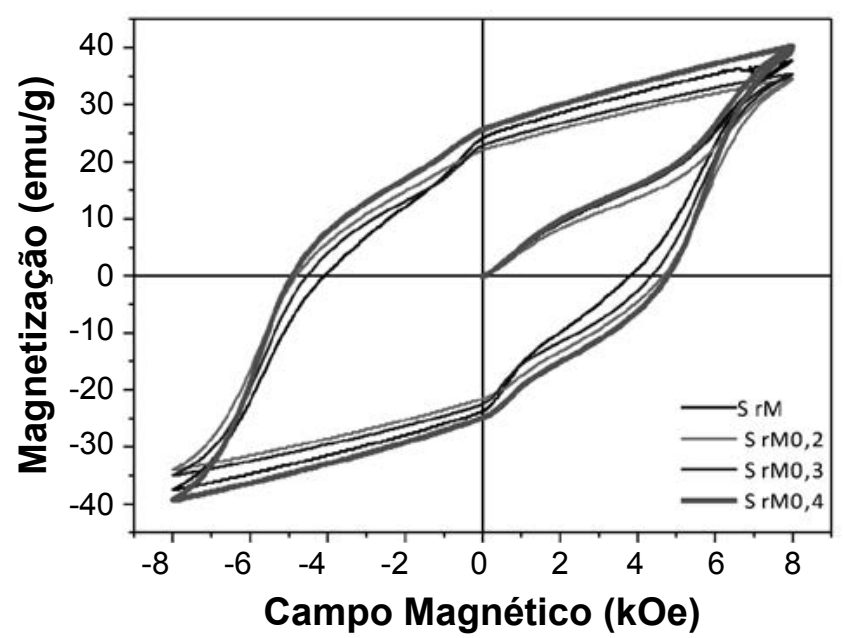

Figura 4: Magnetização como função do campo magnético aplicado à temperatura ambiente dos pós de hexaferritas de estrôncio dopadas com cromo.

[Figure 4: Magnetization as a function of applied magnetic field of chromium-doped strontium hexaferrite powders measured at room temperature.]
Tabela II - Parâmetros magnéticos dos pós sintetizados por reação de combustão.

[Table II - Magnetic parameters of powders obtained by combustion reaction.]

\begin{tabular}{ccccc}
\hline & SrM & SrM0,2 & SrM0,3 & SrM0,4 \\
\hline $\mathrm{Ms}(\mathrm{emu} / \mathrm{g})$ & 37,8 & 34,5 & 35,4 & 40,16 \\
$\mathrm{Mr}(\mathrm{emu} / \mathrm{g})$ & 24,2 & 22,0 & 22,8 & 25,4 \\
$\mathrm{Hc}(\mathrm{kOe})$ & 3,9 & 4,6 & 4,5 & 4,9 \\
\hline
\end{tabular}

os dados reportados [18], nos quais foi mostrado que a dopagem apropriada da hexaferrita de estrôncio está em 0,4 . Isto decorrente ao fato de que a magnetização total da hexaferrita de estrôncio dopada com cromo é originada pelos momentos magnéticos dos íons de cromo que substituirão os íons de ferro na rede. $\mathrm{O}$ íon $\mathrm{Cr}^{3+}$ cujo momento magnético de Bohr é $3 \mu \mathrm{B}$, inferior ao do $\mathrm{Fe}^{3+}$ cujo momento magnético de Bohr é $5 \mu \mathrm{B}$, o aumento da magnetização quando $\mathrm{x}=0,4$ pode ser atribuído a uma ocupação de íons $\mathrm{Cr}^{3+}$ nos sítios octaedrais $4 \mathrm{f} 2$ (spin down) maior, do que nos sítios octaedrais $12 \mathrm{~K}$ e $2 \mathrm{a}$ (spin up), ocasionando um aumento do momento magnético total da rede. Por outro lado, as características extrínsecas como tamanho de grão e/ou partícula influenciam a área de domínio magnético e podem contribuir para o aumento da magnetização, pois quanto maior o tamanho da partícula e/ou dos grãos, menor o número de barreiras, favorecendo uma maior magnetização. Neste trabalho, foi verificado que a coercividade $(\mathrm{Hc})$ variou com a dopagem de cromo, pois o aumento do teor de cromo na hexaferrita de estrôncio favoreceu o aumento do campo coercitivo $(\mathrm{Hc})$ e a diminuição da fase intermediária pela redução do tamanho de partículas, como foi apresentado na Tabela I. Estes resultados concordam com resultados reportados [15, 19, 20]. Assim, ficou evidente a influência do cromo em substituição aos íons de ferro na rede da hexaferrita de estrôncio tanto na síntese, quanto nas características morfológicas e magnéticas dos pós sintetizados por reação de combustão.

\section{CONCLUSÕES}

A síntese por reação de combustão foi favorável para a obtenção de pós cristalinos de hexaferritas de estrôncio dopadas com cromo. Todas as composições estudadas apresentaram a formação da fase cristalina hexagonal da magnetoplumbita, e traços de fase secundária $\left(\mathrm{SrFe}_{2} \mathrm{O}_{5}\right)$. O aumento da concentração de cromo interferiu diretamente nas características estruturais das partículas, causando aumento do campo coercivo. As hexaferritas de estrôncio estudadas $\mathrm{SrCr}_{\mathrm{x}} \mathrm{Fe}_{12-\mathrm{x}} \mathrm{O}_{19}, \mathrm{x}=0,0,2,0,3$ e $0,4 \mathrm{~mol} \% \mathrm{de} \mathrm{Cr}^{3+}$ apresentaram características magnéticas diferenciadas, de acordo com a quantidade de dopante utilizado, permitindo o uso desses materiais como magnéticos duros, com ciclos de magnetização relativamente largos.

\section{AGRADECIMENTOS}

À FAPESP pelo apoio financeiro (Procs. 2008/040250 e 2012/24025-0), aos alunos Leonardo José Dalla Costa 
e Ricardo José da Silva Afonso pelos ensaios magnéticos, à CAPES pela bolsa concedida e ao programa de Pósgraduação em Ciência e Engenharia de Materiais da Universidade Federal de S. Carlos.

\section{REFERÊNCIAS}

[1] S. E. M. Ghahfarokhi, F. Ranjbar, J. Mag. Mag. Mater. 349 (2013) 128-133.

[2] S. Alamolhoda, S. Ebrahimi, A. Badiei, Phys. Metals 102 (2006), 71-73.

[3] S. Kanagesan, S. Jesurani, R. Velmurugan, Sci. Res. 23 (2012) 1511-1514.

[4] Q. Yang, H. Zhang, Y. Liu, Q. Wen, Mater. Lett. 63 (2008) 406-408.

[5] M. Yasuda, L. Hasegawa, M. R. Morelli, Cerâmica 53, 328 (2007) 404-410.

[6] M. Iqbal, M. Ashiq, I. Gul, J. Mag. Mag. Mater. 322 (2010) 1720-1726

[7] J. Wang, C. Ponton, I. Harris, J. Alloys Comp. 403 (2005) 104-109.)

[8] A. C. F. M. Costa, A. P. Diniz, V. J. Silva, H. S. Ferreira, A. A. Costa, D. Cornejo, R. H. G. A. Kiminami, L. Gama, J. Alloy. Compnd. 483 (2009) 655-657.

[9] A. C. F. M. Costa, A. P. Diniz, A. G. B. de Melo, R. H.
G. A. Kiminami, D. Cornejo, A. A. Costa, L. Gama, J. Mag. Mag. Mater. 320 (2008) 742-749.

[10] A. C. F. M. Costa, M. Morelli, R. H. G. A. Kiminami, "Combustion Synthesis Processing of Nanoceramics", Handbook of Nanoceramics and their Based Nanodevices, Ed. Am. Sci. Publ. (2009) cap. 80.

[11] S. M. Masoudpanah, S. A. Ebrahimi, J. Mag. Mag. Mater. 342 (2013) 128-133.

[12] L. Gama, P. Hernandez, J. Mag. Mag. Mat. 317 (2007) 29-33.

[13] S. Ounnunkad, P. Winotai, J. Mag. Mag. Mater. 301 (2006) 292-300.

[14] A. C. F. M. Costa, P. Sarubo-Jr, D. A. Vieira, V. J. Silva, T. S. Barros, D. R. Cornejo, R. H. G. A. Kiminami, Cerâmica 55, 333 (2009) 78-83.

[15] Z. Jing, W. Tang, J. Mag. Mag. Mater. 295 (2005) 263268.

[16] J. S. Reed, "Principles of Ceramics Processing", John Wiley, New York, EUA (1996).

[17] A. P. Guimaraes, "Magnetism and Magnetic Resonance in Solids", John Wiley, New York, EUA (1998) 297.

[18] Q. Fang, H. Cheng, J. Mag. Mag. Mater. 294 (2005) 28-286.

[19] A. Abbas, N. Mohsen, K. Mackenzie, J. Mater. Sci: Mater. Elet. 22 (2011) 1297-1302.

(Rec. 28/01/2014, Rev. 17/02/2014, Ac. 18/02/2014) 